\title{
Protocol for evaluation of perioperative risk in patients aged over 75 years: Aged Patient Perioperative Longitudinal Evaluation-Multidisciplinary Trial (APPLE- MDT study)
}

Yanhong Zhang ${ }^{1,2}$, Lina Ma ${ }^{3}$, Tianlong Wang ${ }^{4}$, Wei Xiao ${ }^{4}$, Shibao Lü ${ }^{5}$ Chao Kong ${ }^{5}$, Chaodong Wang ${ }^{2}$, Xiaoying Li ${ }^{1}$, Yun Li ${ }^{3}$, Chunlin Yin ${ }^{6}$, Suying Yan ${ }^{7}$, Ying Li $i^{8}$, Kun Yang ${ }^{9}$, Piu Chan ${ }^{2,10,11^{*}}$ and APPLE-MDT Study Working Group

\begin{abstract}
Background: With the extended life expectancy of the Chinese population and improvements in surgery and anesthesia techniques, the number of aged patients undergoing surgery has been increasing annually. However, safety, effectiveness, and quality of life of aged patients undergoing surgery are facing major challenges. In order to standardize the perioperative assessment and procedures, we have developed a perioperative evaluation and auxiliary decision-making system named "Aged Patient Perioperative Longitudinal Evaluation-Multidisciplinary Trial (APPLE-MDT)".
\end{abstract}

Methods: We will conduct a perioperative risk evaluation and targeted intervention, with follow-ups at 1, 3, and 6 months after surgery. The primary objective of the study is to evaluate the effectiveness of the "Aged Patient Perioperative Longitudinal Evaluation-Multiple Disciplinary Trial Path" (hereinafter referred to as the APPLE-MDT path) in surgical decision-making for aged patients ( $\geq 75$ years) undergoing elective surgery under non-local anesthesia in the operating room. The secondary objectives of the study are to evaluate the postoperative outcome and health economics of the APPLE-MDT path applied to the surgical decision-making of aged patients ( $\geq 75$ years) undergoing elective surgery under non-local anesthesia and to optimize intervention strategies for aged patients undergoing surgery to reduce the occurrence of postoperative complications and improve the quality of life after surgery.

\footnotetext{
* Correspondence: pbchan90@gmail.com

${ }^{2}$ China National Clinical Research Center for Geriatric Disorders, Beijing 100053, China

${ }^{10}$ Department of Neurology and Neurobiology, Xuanwu Hospital of Capital Medical University, Beijing 100053, China

Full list of author information is available at the end of the article
}

(C) The Author(s). 2021 Open Access This article is licensed under a Creative Commons Attribution 4.0 International License, which permits use, sharing, adaptation, distribution and reproduction in any medium or format, as long as you give appropriate credit to the original author(s) and the source, provide a link to the Creative Commons licence, and indicate if changes were made. The images or other third party material in this article are included in the article's Creative Commons licence, unless indicated otherwise in a credit line to the material. If material is not included in the article's Creative Commons licence and your intended use is not permitted by statutory regulation or exceeds the permitted use, you will need to obtain permission directly from the copyright holder. To view a copy of this licence, visit http://creativecommons.org/licenses/by/4.0/. The Creative Commons Public Domain Dedication waiver (http://creativecommons.org/publicdomain/zero/1.0/) applies to the data made available in this article, unless otherwise stated in a credit line to the data. 
(Continued from previous page)

Discussion: It is necessary to formulate a reliable, effective, and concise evaluation tool, which can effectively predict the perioperative complications and mortality of aged patients, support targeted intervention strategies, and allow for a more comprehensive risk and benefit analysis, thereby forming an effective senile perioperative surgery management path. It is expected that the implementation of this protocol can reduce the occurrence of postoperative complications, improve the postoperative quality of life, shorten hospital stay, reduce hospitalization expenses, reduce social burden, and allow the elderly to have a good quality of life after surgery.

Trial registration: ChiCTR, ChiCTR1800020363, Registered 15 December 2018.

Keywords: Advanced-age patients, Perioperative assessment, Age-related surgical risk factors, Multidisciplinary evaluation

\section{Background}

The proportion of the world population aged $\geq 60$ years will increase from $11.0 \%$ in 2010 to $21.8 \%$ in 2050 while the portion of aged $\geq 80$ years will increase from 1.5 to $4.3 \%$ [1]. Aged patients, especially those over 75 years, more likely suffer from problems such as frailty, geriatric syndromes, comorbidities, polypharmacy, decline of organ function, and deteriorating brain health. As a result, the operability of aged patients decreases. Moreover, the risk of postoperative complications is significantly higher than that of other patients, and the rehabilitation process after surgery is slow. As a result, the safety, effectiveness and quality of life of aged patients undergoing surgery are facing major challenges.

In recent years, with an increase of aged patients undergoing surgery, perioperative management has become the focus of scientific research. Surgeons and anesthetists have gradually realized the importance of multidisciplinary comprehensive evaluation of aged patients undergoing surgery. The comprehensive geriatric assessment (CGA) is defined as a multidimensional and interdisciplinary process, which focuses on determining the medical, psychological, and functional capabilities of the frail elderly to formulate a coordinated and comprehensive treatment and longterm follow-up plan [2]. Since elderly patients often have multiple pathologies, preoperative assessment frequently calls for a multidisciplinary approach applying the concepts of CGA to guide the patient through the perioperative period [3]. Currently studies have found that the components of CGA can be used as predictors of postoperative complications among geriatric patients undergoing surgery. CGA results showed that patients with adverse outcomes were associated with functional dependency and poor nutrition $[4,5]$. The cumulative number of impairments in the CGA domains was significantly associated with adverse outcomes, in-hospital events, and prolonged hospital stays [4]. Aged patients undergoing intervention after evaluation result in shorter hospital stay and reduced mortality [6]. It is suggested that multidisciplinary perioperative management plays an important role in elderly patients undergoing surgery.

However, perioperative management of the elderly is a complicated process, which needs more evidence to support best practices. The quantity of clinical trials available is really miserable. Some studies have shown that the complexity of CGA causes certain limitations in application [7]. Although CGA as a measure of preoperative evaluation are well recognized, there is no gold standard assessment that is universally used in the clinical and research setting.

This study aims to develop a perioperative multidisciplinary evaluation system for aged patients over 75 years undergoing surgery, which could identify and stratify risks, formulate preoperative, intraoperative and postoperative intervention strategies. We, therefore, construct a standardized "Longitudinal Perioperative Evaluation of Aged People (APPLE)" System for perioperative multidisciplinary evaluation and auxiliary decision-making for aged patients, and apply the APPLE-MDT model to guide the perioperative management of aged patients undergoing elective surgery under non-local anesthesia in the operating room, so as to optimize the treatment plan for aged patients and enhance the ability of aged patients to resist external stress with the goal of improving the outcome of such patients.

\section{Methods and design}

\section{Study aim}

This study will aim to develop the APPLE-MDT system and conduct multidisciplinary perioperative evaluation and intervention on the study group using the APPLEMDT auxiliary system.

The primary goal of the perioperative assessment is to develop the perioperative multidisciplinary evaluation path (hereafter referred to as the APPLE-MDT path) in the surgical decision-making and intervention strategies for aged patients ( $\geq 75$ years) undergoing elective surgery under non-local anesthesia in the operating room. 
The secondary objective is to evaluate the effectiveness of the APPLE-MDT path with the postoperative outcome and health economics after applied to the aged patients ( $\geq 75$ years) undergoing elective surgery, so as to reduce the occurrence of postoperative complications and improve the quality of postoperative life.

\section{Study design and setting}

This study is a single-center, parallel, randomized, controlled study. This study will be carried out in Xuanwu Hospital, Capital Medical University, which is a tertiary teaching hospital in China. The control group will undergo anesthesia and surgery according to an established clinical routine. The APPLE-MDT information system will be used for the APPLE-MDT group to assist in perioperative clinical decision-making by identifying risks and providing suggestions for mitigating interventions to be carried out. Preoperative evaluation will be completed within $48 \mathrm{~h}$ after the patient is admitted to hospital. Follow up assessments will be conducted at 1 , 3 , and 6 months after surgery. The multidisciplinary team will include specialists in surgery, anesthesiology, geriatrics, neurology, cardiology, pharmacy, nutrition, nursing, and rehabilitation. The detailed study flow chart is shown in Fig. 1.

The study started in March 2019. The recruitment period will be 30 months, with a follow-up of 6 months. The results of the study will be expected in mid-2022.

\section{Randomization}

Patients over 75 years scheduled for non-cardiac surgery under non-local anesthesia, who meet pre-specified eligibility criteria, will be enrolled and randomized into the control group or the APPLE-MDT group by computer software. The randomization scheme will be blinded by the complete randomization scheme blinding method. Neither the subjects nor the follow-up personnel will know the grouping.

\section{Intervention scheme}

Two groups will be planned for this study.

Control group: Participants enrolled will receive anesthesia, surgery, and postoperative rehabilitation according to an established clinical routine.

APPLE-MDT group (Fig. 2) will include:

1) Preoperative assessment (APPLE-MDT path): A comprehensive multidisciplinary evaluation on 15 categories and 25 scales will be developed according to the recommendations of guidelines and consensus as well as consultation with the specialists [8-19] (Table 1). The APPLE-MDT system will automatically extract abnormal values on the relevant scales, identify likely perioperative risks, and recommend the comprehensive management opinions for patients. The multidisciplinary team will finally decide and conduct targeted intervention according to the APPLE-MDT system.

2) Intraoperative intervention: Following consideration of perioperative risks, the anesthetist group will implement individualized anesthesia schemes for each patient including full sedation, analgesia, antistress management, accurate monitoring indicator management, objectives-oriented liquid management, and circulation ventilation management, as appropriate.

3) Post-operative intervention: The acute pain service (APS) group will provide individualized analgesic service. Depending on the patients' condition, the patients will be transferred to a specialized ward, geriatric comorbidity ward, or an ICU for diagnosis and treatment. Enhanced recovery after surgery will be carried out, and the rehabilitation process and postoperative variables of the patients will be recorded. Multidisciplinary evaluation will be conducted again before the patients are discharged; according to the evaluation results, rehabilitation plans (including the combined medication plan, nutritional state health guidance, functional state guidance plan, fall prevention plan, and rehabilitation precautions given by specialties, as required) will be formulated.

4) Follow-up: All subjects enrolled will undergo examination of postoperative complications, specialist follow-ups, and follow-ups of long-term quality of life (ADL, IADL) at 1, 3, and 6 months after surgery, respectively. In addition, an assessment of frailty (the Fried frailty phenotype scale) will be conducted at 6 months after surgery. The follow-up variables will be recorded. The follow-up assessments will be conducted during the patients' hospital visits. Relevant web pages and mobile phone applications will be developed as remedial measures in case a follow-up cannot be conducted on site.

\section{Study outcomes}

Primary outcomes: Incidence of non-specialty severe systemic complications (acute stroke, postoperative delirium, acute respiratory failure, acute heart failure, acute myocardial injury and acute renal injury) and all-cause mortality over the first 30 days.

Secondary outcomes: (1) Quality of life score, severe complications of certain specialties, anesthesia complications, complications of concomitant medication, performance status, functional rehabilitation of certain specialties, and postoperative rehabilitation process 


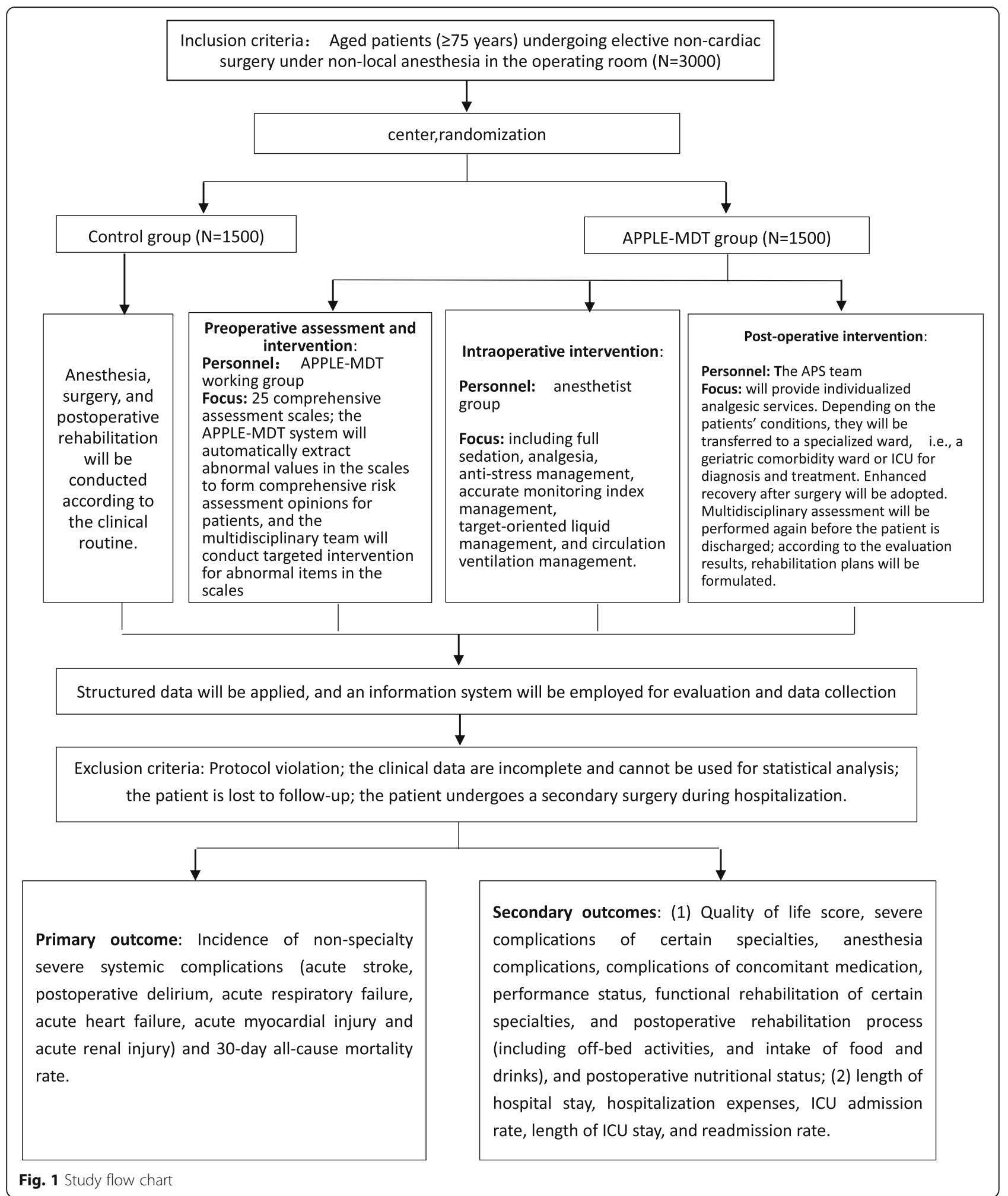

(including off-bed activities, intake of food and drinks, etc.), and postoperative nutritional status; (2) Length of hospital stay, hospitalization expenses, ICU admission rate, length of ICU stay, and readmission rate.

\section{Inclusion criteria}

Patients are eligible for the study if they and their families agree with this protocol and sign an informed consent form. The patients must cooperate with the 


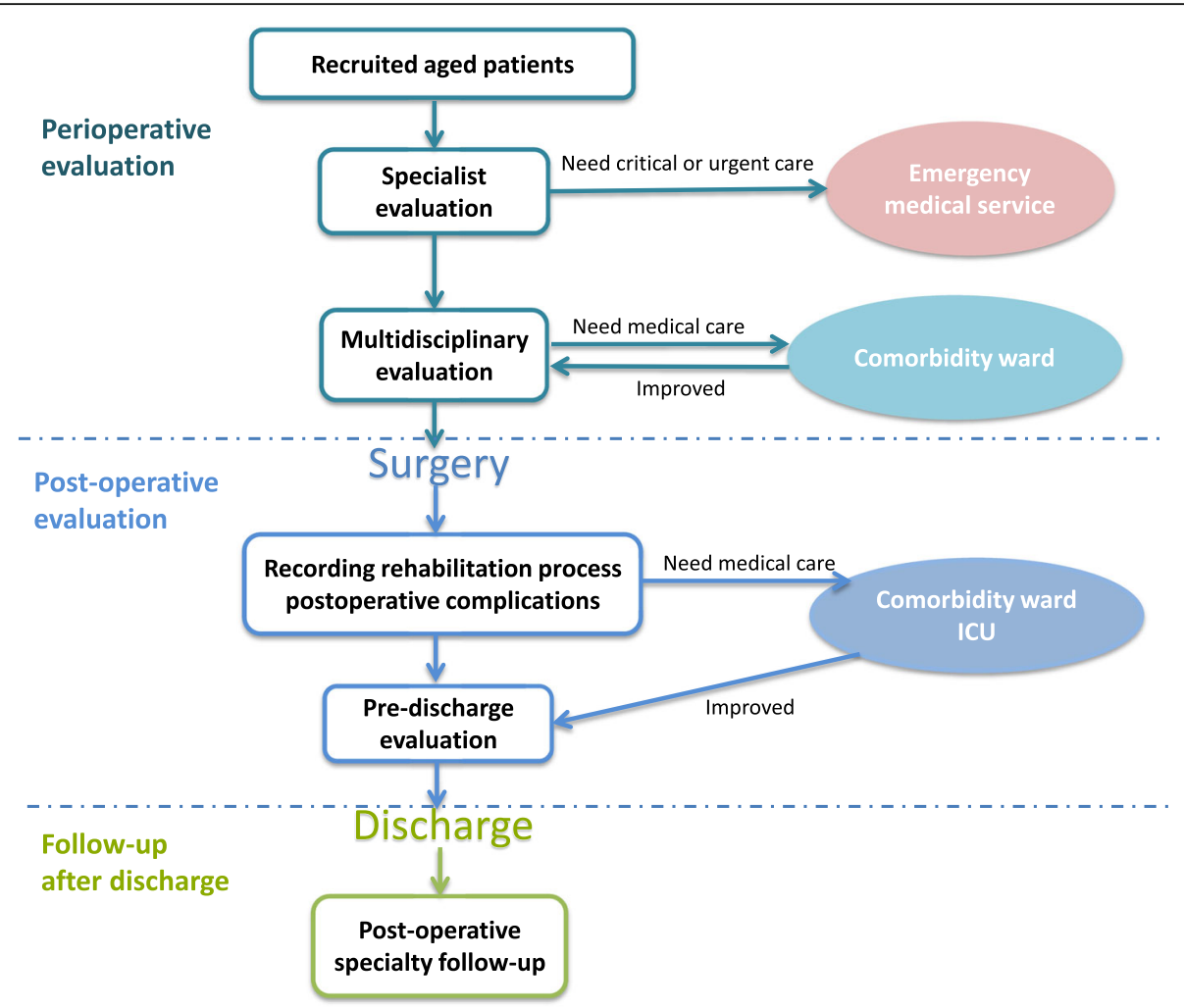

Fig. 2 Aged Patient Perioperative Longitudinal Evaluation-Multidisciplinary Clinical Research (APPLE-MDT) clinical path

evaluation, have no contraindications to surgery, be over 75 years, not be undergoing local anesthesia, and not be undergoing elective cardiac surgery.

\section{Exclusion criteria}

Patients are not eligible for the study if they are aged below 75 years; undergoing emergency or day surgery; undergoing surgery under local anesthesia; have an urgent condition that needs to be managed before the surgery, are unable to cooperate with the evaluation; they or their families refuse their participation in the study.

If a patient enrolled in the study requires a secondary surgery during hospitalization, the treatment will be carried out according to the diagnosis and established relevant routine; however, the subject will be excluded. If a subject misses any follow-up appointments or necessary clinical data cannot be obtained during the follow-up, the subject will be regarded as lost to follow-up.

\section{Sample size calculation}

According to the literatures, the incidence of perioperative complications in the elderly is $20-28 \%$ [20-23]. It is expected that the APPLE-MDT group will reduce the incidence of complications by $20 \%$ compared with the control group. The APPLE-MDT group and the control group are matched by $1: 1$, assuming a power of $90 \%$ and a two-sided significance level (alpha) of 0.05, the sample size is demonstrated in Figure S1. It is estimated that the incidence of perioperative complications of the elderly in China is $26 \%$, the sample size is 2776 . Considering the loss of follow-up rate is $10 \%, 3054$ participants will be randomized at a ratio of 1:1 into two groups. To establish a cohort, it is assumed that the number of clinical end points in one year is $10-20$ times than the variables included in the model (20 variables). In this study, the incidence of end-point events was about $26 \%$, considering the loss of follow-up rate is $20 \%$, so 1847 cases are calculated. Therefore, 3054 patients recruited can meet the design requirements.

\section{Data collection, validation, and management}

During the study, clinical monitors will regularly check the extracted data to ensure consistency with the contents of electronic medical records. Investigators in the clinical study will receive unified training to ensure standardization of data entry. The investigator should record the contents in the forms truthfully, carefully, and in detail according to the requirements of the study protocol to ensure the accuracy and reliability of the contents in the study medical records. All observations and results of laboratory examination will be verified to ensure the reliability of data and of all conclusions 
Table 1 Multidisciplinary Comprehensive Geriatric Assessment Scales

\begin{tabular}{|c|c|c|c|c|}
\hline \multirow[t]{2}{*}{ SYSTEM } & \multirow[t]{2}{*}{ SCALE } & \multicolumn{3}{|l|}{ EVALUATION PERIOD } \\
\hline & & pre-operative evaluation & $\begin{array}{l}\text { post-operative } \\
\text { evaluation }\end{array}$ & $\begin{array}{l}\text { follow-up at } \\
6 \text { months }\end{array}$ \\
\hline Nutrition & Mini-Nutritional Assessment(MNA) & $x$ & $x$ & $x$ \\
\hline Functional Status & $\begin{array}{l}\text { Activities of Daily Living Scale (ADL) } \\
\text { Instrumental Activities of Daily Living Scale (IADL) }\end{array}$ & $\times$ & $x$ & $x$ \\
\hline Frailty & $\begin{array}{l}\text { Fried Frailty Phenotype } \\
\text { Frailty Screening Questionnaire (FSQ) }\end{array}$ & $\times$ & $\times$ & $\times$ \\
\hline Risk of Fall & Morse Fall Risk Assessment Scale & $x$ & $x$ & \\
\hline Pain & Visual Analogue Scale (VAS) & $x$ & $x$ & \\
\hline Respiratory System Risk Assessment & $\begin{array}{l}\text { Arozullah Postoperative Respiratory Failure } \\
\text { Predictive Score Scale } \\
\text { Preoperative STOP-BANG Screening Diagnosis } \\
\text { of Obstructive Respiratory Sleep Syndrome } \\
\text { Predictive index of lung function for airway } \\
\text { complications and mortality risk after operation }\end{array}$ & $x$ & & \\
\hline Cardiac Risk Assessment & $\begin{array}{l}\text { Cardiac evaluation in accordance with ACC/AHA } \\
\text { Algorithm for non-cardiac surgery }\end{array}$ & $x$ & & \\
\hline Cerebrovascular Risk Assessment & $\begin{array}{l}\text { Modified Framingham Stroke Risk Score } \\
\text { Essen Stroke Risk Score }\end{array}$ & $x$ & & \\
\hline Liver Function and Renal Function & Medical history and laboratory test & $x$ & & \\
\hline Blood Glucose & Laboratory test & $x$ & & \\
\hline Cognitive Function & $\begin{array}{l}\text { Mini-mental state examination (MMSE) } \\
\text { Montreal Cognitive Assessment (MoCA) } \\
\text { Risk Factors for Postoperative Delirium }\end{array}$ & $x$ & $\times$ & \\
\hline Anxiety and Depression & $\begin{array}{l}\text { Self-rating Anxiety Scale (SAS) } \\
\text { Self-rating Depression Scale (SDS) }\end{array}$ & $x$ & $\times$ & \\
\hline Thrombosis and Hemorrhage & $\begin{array}{l}\text { Caprini Thrombosis Risk Factor Assessment } \\
\text { Risk Assessment of Hemorrhagic Complications }\end{array}$ & $\times$ & & \\
\hline Polypharmacy & $\begin{array}{l}\text { detailed medication history } \\
\text { Appropriate perioperative adjustments }\end{array}$ & $x$ & $\times$ & \\
\hline Anesthesia-related Assessment & $\begin{array}{l}\text { ASA } \\
\text { Airway-related Assessment } \\
\text { Intraspinal -related Assessment }\end{array}$ & $x$ & & \\
\hline
\end{tabular}

derived from this data. All data in this study will be collected automatically by the purpose-developed system. A patent will be applied for the system design scheme. The scores and formulas will be calculated based on the patients' original data.

\section{Statistical analysis}

Data will be presented as mean \pm SD or mean rank or number and percentage. The difference in the characteristics between the two groups will be evaluated by chisquared test for categorical variable, independent t-test for continuous variables, and Kruskal-Wallis comparisons for the abnormally distributed continuous variables, with adjustment for potential confounders by multivariable regression analysis. A Cox proportional hazards model was used to evaluate the effect of covariates on all-cause mortality. We considered $p<0.05$ (two-tailed) statistically significant. Statistical analyses were performed with SPSS (Chicago, IL, USA, version 11.0).

\section{Discussion}

Elderly patients undergo surgical interventions 4 times more often than the rest of the population [24]. The aging process determines a reduction of physiological reserve, which means that elderly person may become more vulnerable to stressors [25]. The prevalence of comorbidities and frailty is high. This aging process and clinical complexity especially creates a specific status that can modify the response to operations. This fact should prompt us to seek accurate clinical study that is specific to this age-group, so that there are reduced postoperative complications and mortalities for aged patients undergoing surgery.

When it comes to perioperative management of elderly patients, preoperative evaluation that can stratify risks and assist decision-making is very important.

The assessment of the surgical risk is currently performed by different instruments. Physiological and Operative Severity Score for the Enumeration of Mortality 
and Morbidity (POSSUM) [26], Surgical Risk Score (SRS) [27] that includes the National Confidential Enquiry into Patient Outcome of Death (NCEPOD) [28], American Society of Anesthesiologists (ASA) [29], and British United Provident Association (BUPA) are the more validated ones [30]. These scales are applied at any age, so they don't take into account the characteristics of the elderly, such as frailty, functional declines.

CGA ensures that clinical and functional problems are identified, quantified, and managed appropriately by involving a multidisciplinary team [31]. There is literature demonstrates CGA can predict a higher risk of adverse outcomes independent of established surgical risk assessment tools [23]. The American College of Surgeons (ACS) recognizes the necessity for quality improvement in the surgical care of geriatric patients with the recent publication of guidelines for preoperative assessment in this population, including frailty assessment, and multiple components of the CGA (e.g., depression screening, nutritional status, cognitive ability, and functional status) [11]. Recently, significant improvements have been established in the perioperative management of hip fracture in aged patients. The effective collaboration between geriatrics and traumatology with the implementation of the orthogeriatric units have improved the outcomes [32]. Meta-analysis of orthopedic treatment after hip fracture has shown that the mortality rate and length of hospital stay following the surgery are relatively low [33]. However, preoperative evaluation of geriatric patients has not been standardized based on large-scale clinical evidence validated. Therefore, Perioperative management for aged patients still suffers from empirical, subjective in most institutions [31].

The purpose of our research is not to "identify" the risks of surgery itself. The objective of this study is to identify relevant medical problems and functional conditions, stratify the perioperative risks, predict potential perioperative problems, formulate optimal intervention plans. Therefore we choose these essential domains referred in guidelines and literatures, which are reliable, validated and brief to determine risk for morbidity and mortality in older patients, thereby forming an effective geriatric perioperative management path.

Traditionally, comprehensive geriatric preoperative assessment is time-consuming. We have developed "APPLE-MDT" information system which can efficiently and objectively collect evaluation data. This study verify the effectiveness of the "APPLE-MDT" perioperative management path, thus providing evidence for the perioperative management of aged patients. As some studies have shown that the risk of postoperative complications and death is not significantly related to the type of surgery [3], we will include patients undergoing different types of surgery in the study. As a result, this study may have a higher generalizability than those of studies performed in patients undergoing a single intervention.

At present, there is a lack of standard path and perioperative management of aged patients in China. We put forward a comprehensive and holistic study protocol that aims at evaluating perioperative risks of aged patients undergoing surgery in China, and further develops standardized strategies. It is expected that the implementation of this protocol can reduce the occurrence of postoperative complications, improve the postoperative quality of life, shorten hospital stay, reduce hospitalization expenses, reduce social burden, and allow the elderly to have a good quality of life after surgery.

\section{Supplementary Information}

The online version contains supplementary material available at https://doi. org/10.1186/s12877-020-01956-3.

Additional file 1: Figure S1. Sample size calculation.

\section{Abbreviations}

APS: Acute pain service; APPLE-MDT: Aged Patient Perioperative Longitudinal Evaluation-Multiple Disciplinary Trial; CGA: Comprehensive geriatric assessment; POSSUM: Physiological and Operative Severity Score for the Enumeration of Mortality and Morbidity; SRS: Surgical Risk Score; NCEPOD: National Confidential Enquiry into Patient Outcome of Death; ASA: American Society of Anesthesiologists; BUPA: British United Provident Association; ACS: the American College of Surgeons

\section{Acknowledgements}

We acknowledge all the people who participated in the APPLE-MDT Study.

\section{Authors' contributions}

$Y Z$ designed the study and wrote the paper with $L M ; T W, W X, S L, C K, X L, Y L$, $C Y, S Y, Y L, C W$ and PC designed the study. KY performed statistical analysis. All authors read and approved the final manuscript.

\section{Funding}

Beijing Hospitals Authority's Cultivation Plan (PG2019017). The funder of the study has no role in study design, data collection, data analysis, data interpretation, or writing the manuscript.

\section{Availability of data and materials \\ Not applicable.}

\section{Ethics approval and consent to participate}

This study was approved by the ethics review board of Xuanwu Hospital of Capital Medical University (2018-086) and all the participants provided written informed consent.

\section{Consent for publication \\ Not applicable.}

\section{Competing interests}

The authors declare that they have no competing interests.

\section{Author details}

${ }^{1}$ Medical Administration Division, Xuanwu Hospital of Capital Medical University, Beijing 100053, China. ${ }^{2}$ China National Clinical Research Center for Geriatric Disorders, Beijing 100053, China. ${ }^{3}$ Department of Geriatrics, Xuanwu Hospital of Capital Medical University, Beijing 100053, China. ${ }^{4}$ Department of Anesthesiology, Xuanwu Hospital of Capital Medical University, Beijing 100053, China. ${ }^{5}$ Department of Orthopaedics, Xuanwu Hospital of Capital Medical University, Beijing 100053, China. ${ }^{6}$ Department of Cardiology, Xuanwu Hospital of Capital Medical University, Beijing 100053, China. 
${ }^{7}$ Department of Pharmacy, Xuanwu Hospital of Capital Medical University, Beijing 100053, China. ${ }^{8}$ Department of Nutrition, Xuanwu Hospital of Capital Medical University, Beijing 100053, China. ${ }^{9}$ Department of Evidence-based Medicine, Xuanwu Hospital of Capital Medical University, Beijing 100053, China. ${ }^{10}$ Department of Neurology and Neurobiology, Xuanwu Hospital of Capital Medical University, Beijing 100053, China. "'Department of Neurology, Xuanwu Hospital of Capital Medical University, Beijing 100053, China.

Received: 20 August 2020 Accepted: 10 December 2020

Published online: 06 January 2021

\section{References}

1. Population Division of the Department of Economic and Social Affairs of the United Nations Secretariat. World population prospects: The 2010 Revision. http://esa.un.org/unpd/wpp/unpp/p2k0data.asp.

2. SletvoldO HJL, Thingstad P, et al. Effect of in-hospital comprehensive geriatric assessment (CGA) in older peoplewith hip fracture. The protocol of the Trondheim Hip Fracture trial. BMC Geriatr. 2011;11:18.

3. Dewan SK, Zheng SB, Xia SJ. Preoperative geriatric assessment: comprehensive, multidisciplinary and proactive. Eur J Int Med. 2012;23:48794.

4. Kim Kl, Park KH, Koo KO, et al. Comprehensive geriatric assessment can predict postoperative morbidity and mortality in elderly patients undergoing surgery. Arch Gerontol Geriatr. 2015;56:507-12.

5. Partridge JS, Harari D, Martin FC, et al. The impact of comprehensive geriatric assessment on postoperative outcomes in older patients undergoing scheduled surgery: a systematic review. Anaesthesia. 2014; 69(Suppl 1):8-16.

6. Harari D, Hopper A, Dhesi J, et al. Proactive care of older people undergoing surgery ('POPS'): designing, embedding, evaluating and funding a comprehensive geriatric assessment service for older elective surgical patients. Age Ageing. 2007;36:190-6.

7. Partridge JSL, Collingridge $\mathrm{G}$, Gordon $\mathrm{AL}$, et al. Where are we in perioperative medicine for older surgical patients? A UK survey of geriatric medicine delivered services in surgery. Age Ageing. 2014;43:721-4.

8. Morley JE, Malmstrom TK, Miller DK. A simple frailty questionnaire (FRAIL) predicts outcomes in middle aged African Americans. J Nutr Health Aging. 2012;16:601-8

9. Katz S, Downs TD, Cash HR, et al. Progress in development of the index of ADL. Gerontologist. 1970;10:20-30.

10. Saporito A, Sturini E. Incidence of postoperative delirium is high even in a population without known risk factors. J Anesth. 2014;28:198-201.

11. Chow WB, Rosenthal RA, Merkow RP, et al. Optimal preoperative assessment of geriatric surgical patient: a best practice guideline of the American College of Surgeons National Surgical Quality Improvement Program and the American geriatric society. J Am Coll Surg. 2012;215:453-66.

12. Griffiths R, Beech F, Brown A, Dhesi J, Foo I, Goodal J, Harrop-Grifiths W, Jameson J, Pappenheim K, White S. Guidelines: perioperative care of the elderly. Anaesthesia. 2014;69(Suppl. 1):81-98.

13. Mohanty S, Rosenthal RA, Russel MM, et al. Optimal perioperative management of the geriatric patient: a best practices guideline from the American College of Surgeons NSQIP and the American Geriatrics Society. J Am Coll Surg. 2016;222:930-47.

14. American Geriatrics Society Expert Panel on Postoperative Delirium in Older Adults. Postoperative delirium in older adults: best practice statement from the American Geriatrics Society. J Am Coll Surg. 2015;220(2):136-48.e1.

15. Aldecoa C, Bettelli G, Bilotta F, et al. European Society of Anaesthesiology evidence-based and consensus-based guideline on postoperative delirium. Eur J Anaesthesiol. 2015:34:192-214.

16. Kim S, Brooks AK, Groban L. Preoperative assessment of the older surgical patient: honing in on geriatric syndromes. Clin Interv Aging. 2017;10:13-27.

17. Sattar $\mathrm{S}$, Alibhai $\mathrm{S}$, Wildiers $\mathrm{H}$, et al. How to implement a geriatric assessment in your clinical practice. Oncologist. 2014;19:1056-68.

18. Kristensen SD, Knuuti J, Saraste A, et al. 2014 ESC/ESA guidelines on noncardiac surgery: cardiovascular assessment and management. The joint task force on noncardiac surgery: cardiovascular assessment and management of the European Society of Cardiology (ESC) and the European Society of Anaesthesiology (ESA). Eur Heart J. 2014;35:2383-31.

19. Fleisher LA, Fleischmann KE, et al. ACC/AHA guideline on perioperative cardiovascular evaluation and Management of Patients Undergoing Noncardiac Surgery. J Am Coll Cardiol. 2014;64:e77-e137.
20. Liu LL, Leung JM. Predicting adverse postoperative outcomes in patients aged 80 years or older. J Am Geriatr Soc. 2000;48:405-12.

21. Turrentine FE, Wang $\mathrm{H}$, Simpson VB, et al. Surgical risk factors, morbidity, and mortality in older patients. J Am Coll Surg. 2006;203:865-77.

22. Hamel MB, Henderson WG, Khuri SF, et al. Surgical outcomes for patients aged 80 and older: morbidity and mortality from major noncardiac surgery. J Am Geriatr Soc. 2005;53:424-9.

23. Abete $P$, Cherubini A, Di Bari $M$, et al. Does comprehensive geriatric assessment improve the estimate of surgical risk in elderly patients? An Italian multicenter observational. Am J Surg. 2016;211:76-83.

24. Richardson JD, Cocanour CS, Kern JA, et al. Perioperative risk assessment in older and high-risk patients. J Am Coll Surg. 2004;199:133-46.

25. Troncale JA. The aging process. Physiologic changes and pharmacologic implications. Postgrad Med. 1996;99:111-4 120-2.

26. Copeland GP, Jones D, Walters M. POSSUM: a scoring system for surgical audit. Br J Surg. 1991;78:355-60.

27. Sutton $R$, Bann $S$, Brooks $M$, et al. The surgical risk scale as an improved tool for risk-adjusted analysis in comparative surgical audit. Br J Surg. 2002;89: 763-8.

28. Hoile RW. The National Confidential Enquiry into Peri-operative Deaths (NCEPOD). Aust Clin Rev. 1993;13(1):11-5.

29. Saklad M. Grading of patients for surgical procedures. Anesthesiology. 1941; 2:281-4.

30. British United Provident Association Ltd (BUPA). Schedule of fees. BUPA Newsl Consultants. 1990;3:3.

31. Pilleri A, Scorcu G. New assessment for advanced age: Italian study protocol on the assessment of surgical risk in the over-75-year age-group. Monaldi Arch Chest Dis. 2017:87:57-60.

32. Della Rocca GJ, Moylan KC, Crist BD, et al. Comanagement of Geriatric Patients With Hip Fractures: A Retrospective, Controlled, Cohort Study. Geriatr Orthop Surg Rehabil. 2013;4(1):10-5.

33. Grigoryan $\mathrm{KV}$, Javedan $\mathrm{H}$, Rudolph JL. Orthogeriatric care models and outcomes in hip fracture patients: a systematic review and meta-analysis. J Orthop Trauma. 2014;28:49-55.

\section{Publisher's Note}

Springer Nature remains neutral with regard to jurisdictional claims in published maps and institutional affiliations.

Ready to submit your research? Choose BMC and benefit from:

- fast, convenient online submission

- thorough peer review by experienced researchers in your field

- rapid publication on acceptance

- support for research data, including large and complex data types

- gold Open Access which fosters wider collaboration and increased citations

- maximum visibility for your research: over $100 \mathrm{M}$ website views per year

At BMC, research is always in progress.

Learn more biomedcentral.com/submissions 